\title{
Spectral Dependence of Coherent Backscattering of Light in a Narrow-Resonance Atomic System
}

D. V. Kupriyanov

I. M. Sokolov

N. V.Larionov

P. Kulatunga

Old Dominion University

C. I. Sukenik

Old Dominion University, csukenik@odu.edu

See next page for additional authors

Follow this and additional works at: https://digitalcommons.odu.edu/physics_fac_pubs

Part of the Atomic, Molecular and Optical Physics Commons

\section{Repository Citation}

Kupriyanov, D. V.; Sokolov, I. M.; Larionov, N. V.; Kulatunga, P.; Sukenik, C. I.; Balik, S.; and Havey, M. D., "Spectral Dependence of Coherent Backscattering of Light in a Narrow-Resonance Atomic System" (2004). Physics Faculty Publications. 17.

https://digitalcommons.odu.edu/physics_fac_pubs/17

\section{Original Publication Citation}

Kupriyanov, D.V., Sokolov, I.M., Larionov, N.V., Kulatunga, P., Sukenik, C.I., Balik, S., \& Havey, M.D. (2004). Spectral dependence of coherent backscattering of light in a narrow-resonance atomic system. Physical Review A, 69(3), 33801. doi: 10.1103/

PhysRevA.69.033801 
Authors

D. V. Kupriyanov, I. M. Sokolov, N. V. Larionov, P. Kulatunga, C. I. Sukenik, S. Balik, and M. D. Havey 


\title{
Spectral dependence of coherent backscattering of light in a narrow-resonance atomic system
}

\author{
D. V. Kupriyanov, I. M. Sokolov, and N. V. Larionov \\ Department of Theoretical Physics, State Technical University, 195251, St. Petersburg, Russia \\ P. Kulatunga, C. I. Sukenik, S. Balik, and M. D. Havey \\ Department of Physics, Old Dominion University, Norfolk, Virginia 23529, USA
}

(Received 4 April 2003; published 1 March 2004)

\begin{abstract}
We report a combined theoretical and experimental study of the spectral and polarization dependence of near-resonant radiation coherently backscattered from an ultracold gas of ${ }^{85} \mathrm{Rb}$ atoms. Measurements in a $\pm 6 \mathrm{MHz}$ range about the $5 s^{2} S_{1 / 2} \rightarrow 5 p^{2} P_{3 / 2}, F=3 \rightarrow F^{\prime}=4$ hyperfine transition are compared with simulations based on a realistic model of the experimental atomic density distribution. In the simulations, the influence of heating of the atoms in the vapor, magnetization of the vapor, finite spectral bandwidth, and other nonresonant hyperfine transitions are considered. Good agreement is found between the simulations and measurements.
\end{abstract}

DOI: 10.1103/PhysRevA.69.033801

PACS number(s): 42.50.Gy, 42.50.Nn, 32.80.Pj, 34.80.Qb

\section{INTRODUCTION}

Coherent wave scattering effects in disordered media display an extraordinary variety of phenomena which are of both fundamental and practical concern. Of particular interest is that coherent wave scattering shows a broad universality which makes possible a qualitatively similar description for different types of wave excitation in a variety of media. These range, as an illustration, from enhancement of light scattering off the lunar regolith and the rings of Saturn, on the one hand [1], to an explanation of the peculiarities in propagation of waves in the solid Earth on the other [2]. In addition, coherent wave scattering is a useful technique for diagnosing the average properties of scatterers in turbid media and for assessing relatively thin surface layers in biological and mechanical materials [2-4]. Of fundamental scientific importance, coherent wave scattering was first recognized by Anderson [5] in the context of interference of electron wave scattering in conductors. As the scattering mean free path decreases and becomes shorter than a characteristic length on the order of the wavelength, wave diffusion slows as a result of wave interference. The limiting case where diffusion ceases is called strong localization, where the propagating wave becomes spatially localized inside the medium. For electromagnetic radiation [3,4], two recent reports of strong localization have been made, one in the optical regime [6] and the other for microwave radiation [7]. A major long-term and fundamental goal of the research presented here, and of other researchers in the field, is to attain strong localization of light, but in an ultracold atomic vapor.

Quite recently, coherent multiple light scattering has been observed in ultracold atomic gases, which form a unique and flexible medium for fundamental studies and practical applications [8-11]. In all cases, the essential physical mechanisms are due to interferences in multiple wave scattering from the components of the medium; under certain not very stringent conditions, the interferences survive configuration averaging, thus generating macroscopic observables. First observations and initial explanations for electromagnetic radiation were of the so-called coherent backscattering (CBS) cone in disordered media [12-14]. For radiation incident on a diffusive medium, the effect manifests itself as a spatially narrow $(\sim 1 \mathrm{mrad})$ cusp-shaped intensity enhancement in the nearly backwards direction [3,4]. As electromagnetic waves are not scalar, the detailed shape and size of the enhancement depends on the polarization of the incident and detected light. Nevertheless, for classical radiation scattering from a ${ }^{1} S_{0} \rightarrow{ }^{1} P_{1}$ atomic transition, the largest possible interferometric enhancement is to increase the intensity by a factor of 2.

Atomic gases, because they have exceptionally high- $Q$ resonances and because the light scattering properties may be readily modified by light polarization or intensity, atomic density, and applied external fields, represent an interesting and flexible medium in which to study the role of multiple scattering. However, to achieve the full potential of atomic scatterers as a practical medium for such studies, it is necessary to significantly cool the atoms, in order to suppress the dephasing effects of atomic motion. Coherent backscattering interference has, in fact, been measured in ${ }^{85} \mathrm{Rb}$ [8-10] and $\mathrm{Sr}$ [11] and quite successfully modeled for resonant and nearresonant scattering as well [15-18]. Measurements have also been made of the magnetic field dependence of the coherent backscattering line shape [19] and of the time dependence, for a particular geometry, of light scattered in the coherent scattering regime [20]. However, there remains a significant range of physical parameters associated with the various processes which have not yet been fully explored. Among these are the influence of light intensity, nonzero ground-state multipoles such as alignment or orientation, cooperative multiple-atom scattering associated with higher atomic density, and more general geometries for time-dependent studies. In the present report we concentrate our attention on another variable: The dependence of the coherent backscattering enhancement on detuning of the probe beam from exact resonance. It is clear that nonresonant excitation of the atomic sample results in a smaller optical depth (and associated larger transport mean free path) of the medium [15,17]. However, the theoretical and experimental results presented here reveal that other more subtle effects, including far-offresonance optical transitions, heating of the vapor by multiple light scattering, and self-magnetization of the vapor 
during the CBS phase, can have significant effects on the spectral variation of the CBS enhancement.

In the following sections we first present an overview of the physical system, including how atomic samples are prepared and characterized, and a brief review of measurements of coherent backscattering from an atomic vapor. This if followed by a summary of the approach to simulate coherent multiple scattering in an ultracold atomic gas. We then present our experimental and theoretical results, with focus on various mechanisms that can influence the spectral variation of the coherent backscattering enhancement factor.

\section{OVERVIEW OF PHYSICAL SYSTEM}

\section{A. Preparation and description of the ultracold atomic sample}

Preparation of the ultracold atomic ${ }^{85} \mathrm{Rb}$ sample used in the measurements described in this paper has been described in detail elsewhere [10], but for completeness will be briefly reviewed here. The samples are formed in a vapor-loaded magneto-optical trap (MOT) which is operated in a standard six-beam configuration. The trapping laser is detuned a frequency of $-2.7 \gamma$ from resonance, where $\gamma \sim 5.9 \mathrm{MHz}$ is the natural linewidth of the $F=3 \rightarrow F^{\prime}=4$ hyperfine transition in ${ }^{85} \mathrm{Rb}$. Laser light for the MOT is derived from an injectionlocked diode laser (Sanyo DL7140-201), which is slaved to a master laser (Hitachi HG7851G). The master laser is locked to a crossover peak produced in a Doppler-free saturated absorption spectrometer. Laser locking is achieved by dithering the master laser current and demodulating the saturation absorption spectrum with a lock-in amplifier. In order to produce the required light for hyperfine repumping, the slave laser is microwave modulated to produce a sideband at the wavelength corresponding to the $F=2 \rightarrow F^{\prime}=3$ hyperfine transition. Light exiting the slave passes through an acoustooptic modulator (AOM), which is used as an optical switch, and subsequently coupled into a single-mode fiber optic patchcord. The combination of the AOM switching and fiber coupling results in an $\sim 65-\mathrm{dB}$ attenuation of the trapping laser light. After exiting the fiber, the trapping light is split into three beams and sent to the MOT. Each beam contains $\sim 3.3 \mathrm{~mW}$ of light and is retroreflected, generating an average $\sim 19 \mathrm{~mW}$ in the center of the chamber.

In order to ascertain the number and density of confined atoms, we employ absorption and fluorescence imaging. We find that the MOT is not completely spherical $[10,15]$, but rather is somewhat "cigar shaped" having $1 / e^{2}$ Gaussian radii of 1.1 and $1.38 \mathrm{~mm}$, where the radius is defined according to the density distribution $n(r)=n_{0} \exp \left(-r^{2} / 2 r_{0}^{2}\right), n_{0}$ being the peak density. This distribution results in an optical depth through the center of the MOT of about 6 , where the optical depth $b$ is defined as resulting in an attenuation of the incident intensity by a factor $e^{-b}$. We determine the peak optical depth by direct measurement of the spectral profile of the transmitted CBS light intensity through the central region of the MOT and fitting it to the expected Beer's Law absorption line shape for an ultracold atomic resonance. In these measurements, probing of the density takes place when the MOT lasers are off, for they result in a significant excitedstate fraction, decreasing the measured optical depth. For a

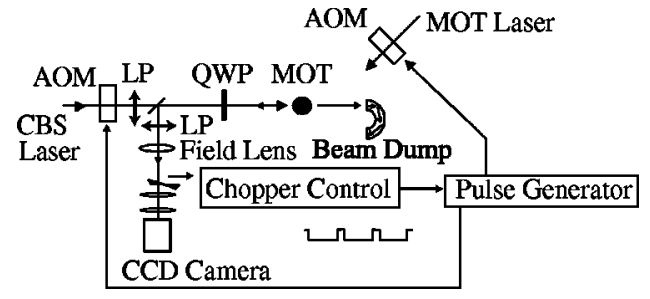

FIG. 1. Schematic diagram of the coherent backscattering apparatus. Shown in the figure is an acousto optic modulator (AOM), magneto-optic trap (MOT), linear polarizer (LP), quarter-wave plate (QWP), and a charge-coupled device (CCD) camera.

Gaussian atom distribution in the MOT, the optical depth is given by $b=\sqrt{2 \pi} \sigma_{0} n_{0} r_{0}$, where $\sigma_{0}$ is the cross section for light scattering [21]. With the values given above and an average Gaussian radius $r_{0}=1.2 \mathrm{~mm}$, we calculate that the MOT contains approximately $4.3 \times 10^{8}$ atoms and has a peak density $n_{0}=1.6 \times 10^{10}$ atoms $\mathrm{cm}^{-3}$. Note that these parameters are large enough to ensure an optical depth large enough for coherent multiple scattering, but that the density is not so large as to necessitate consideration of cooperative pair scattering in the vapor.

The vapor-loaded MOT is formed in a custom-made stainless-steel ultrahigh-vacuum UHV chamber that is pumped by both an ion and titanium sublimation pump. The UHV chamber is fitted with a stainless-steel sidearm containing a valvable and heated $\mathrm{Rb}$ reservoir. Because we are observing light which is backscattered from our sample, it is critical that all other backscattered reflections be suppressed. A major source of unwanted backscattered light is from the vacuum viewports on the MOT chamber. In order to minimize this light, we installed wedged optical quality windows having a "V"-type antireflection (AR) coating at $780 \mathrm{~mm}$ on the probe-laser (described in the following section) entrance and exit ports. The AR coating results in less than $0.25 \%$ reflectivity at $780 \mathrm{~nm}$. Further, the window through which the probe laser beam enters is mounted on a UHV bellows, allowing us to better direct unwanted reflections from entering the charge-coupled device (CCD) detector. We also found it necessary to replace the standard window on the CCD camera with a wedged and near-infrared AR-coated window in order to suppress interference fringe formation in the CCD images.

\section{B. Measurement of atomic coherent backscattering}

We present in this section a brief overview of the coherent backscattering apparatus used to obtain the experimental results reported here. Further details may be found in Kulatunga et al. [10], where the experimental apparatus used in experiments to study coherent radiative transfer in an ultracold gas of ${ }^{85} \mathrm{Rb}[10]$ is described. A schematic diagram of the arrangement is shown in Fig. 1. There the external light source used in the experiment is provided by an external cavity diode laser that is stabilized by saturated absorption to a crossover resonance associated with hyperfine components of the $5 s^{2} S_{1 / 2} \rightarrow 5 p^{2} P_{3 / 2}$ transition. With reference to Fig. 2, which shows relevant hyperfine transitions in ${ }^{85} \mathrm{Rb}$, the laser 


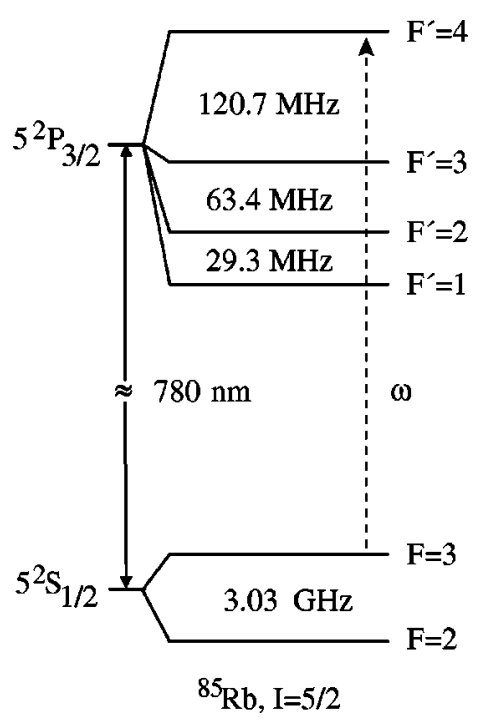
${ }^{85} \mathrm{Rb}$.

may be tuned several hundred $\mathrm{MHz}$ from nearly any hyperfine resonance in ${ }^{85} \mathrm{Rb}$ by a standard offset locking technique using an acousto-optic modulator. Detuning from resonance is defined by $\Delta=\omega_{L}-\omega_{0}$, where $\omega_{L}$ is the CBS laser frequency and $\omega_{0}$ is the $F=3 \rightarrow F^{\prime}=4$ resonance frequency. The laser bandwidth is estimated, from the spectral width of the transmitted intensity of the $F=3 \rightarrow F^{\prime}=2$ transition under weak absorption conditions, to be less than $1 \mathrm{MHz}$. The typical output power is $\sim 5 \mathrm{~mW}$. The laser output is launched into a single-mode polarization preserving fiber and then beam expanded and collimated by a beam expander to a $1 / e^{2}$ diameter of about $8 \mathrm{~mm}$. The polarization of the resulting beam is selected and then the beam passed through a nonpolarizing and wedged 50-50 beam splitter that passes approximately half of the laser power to the atomic sample. The backscattered radiation is directed by the same beam splitter to a field lens of $45 \mathrm{~cm}$ focal length, which condenses the light on the focal plane of a liquid-nitrogen-cooled CCD camera. The diffraction-limited spatial resolution is about $100 \mu \mathrm{rad}$, while the polarization analyzing power is greater than 2000 at $780 \mathrm{~nm}$. There are four polarization channels that are customarily studied in coherent backscattering. For linearly polarized input radiation, two of these correspond to measuring the backscattered light in two mutually orthogonal output channels. This is readily achieved by removing the quarter-wave plate, as shown in Fig. 1, and rotating the linear polarization analyzer located before the field lens. For input radiation of definite helicity - that being generated by the linearly polarized input and the quarter-wave plate-the other two channels correspond to the helicity of the backscattered radiation. This is similarly measured by rotation of the linear polarizer just before the field lens. The instrumentation as described, with some modifications to suppress the intense trapping beam fluorescence, has been previously used to study coherent backscattering in ultracold atomic gases and in solid and liquid samples as well [10].

Measurements of the backscattered light is made by exposing the ultracold atoms to the CBS laser light for an in- terval of $0.25 \mathrm{~ms}$ temporally centered in a 5-ms dark interval during which the MOT lasers are turned off. The MOT lasers are then turned back on for $20 \mathrm{~ms}$, which is sufficiently long that the cold atom sample is reconstituted. This procedure is repeated for $300 \mathrm{~s}$, which constitutes a single experimental run. A run of $300 \mathrm{~s}$ with the MOT absent allows measurement of the background $\mathcal{B}$, which is principally due to hot atom fluorescence excited by the CBS laser. This measurement alone is not sufficient to determine the amount of background contributing during an actual data run. This is because attenuation of the CBS laser by the MOT, during the data-taking phase, reduces the amount of background during the backscattering run, in comparison with the background phase. This is accounted for by auxiliary measurements of the MOT attenuation of the total (spatially integrated) transmitted CBS laser intensity; these measurements are done at each detuning for which data are obtained. As the MOT is very nearly centered in the vacuum chamber, the total hotatom background fluorescence is given by $(\mathcal{B} / 2)(1+C)$, where $C$ is found from measurement of the frequencydependent attenuation of the CBS laser intensity by the MOT. This effect tends to reduce slightly the enhancement deduced from the measurements, compared to values obtained without making the correction. However, the effect is quite small and, within the statistics of the measurements, the same in all four polarization channels and for all detunings used in this experiment. The average shift amounts to less than -0.01 in the enhancement, well within the statistical error in each measurement. Finally, the saturation parameter for the CBS laser is less than $s=0.08$ on resonance, which with the 0.25 -ms measurement interval is sufficient to minimize mechanical action of the CBS laser beam on the atomic sample.

\section{Brief overview of the theoretical treatment}

A general theory of the coherent backscattering process in an ultracold atomic gas has been developed recently by several groups [15-17]. The theoretical development essentially maintains the earlier conceptions of weak localization in the atomic scattering problem [22] and takes into account the influence of the optical depth and sample size on the character of the coherent backscattering cone. In spite of the fact that the basic ladder and interference terms, describing the process, have a similar structure in all the theoretical approaches, there are certain types of accompanying physical phenomena which can become more important as more detailed experimental or theoretical spectral analysis is considered.

In our earlier theoretical approach [15], the general analytical development was realized by a Monte Carlo simulation of coherent multiple scattering in an ultracold $(T<50 \mu \mathrm{K})$ gas of ${ }^{85} \mathrm{Rb}$ atoms confined in a magnetooptical trap. The simulation was closely matched to the experimental density distribution and temperature conditions as described in the previous paragraphs. The radiation field frequency was selected to be in the vicinity of the $F=3 \rightarrow F^{\prime}$ $=4$ hyperfine transition and to have polarization states and a weak-field intensity corresponding to the experimental real- 
ization. The effects of sample size and the spatial and polarization dependence of the coherent backscattering cone were considered in detail. Some aspects of the spectral variation of the coherent backscattering enhancement factor were also considered, including the surprisingly strong influence of the far-off-resonance $F=3 \rightarrow F^{\prime}=3$ and $F=3 \rightarrow F^{\prime}=2$ hyperfine transitions. However, other physical effects can have a profound influence on the spectral variations, and we consider some of those in the present report. Among these, for currently achievable laboratory conditions, are (i) heating of the atomic gas by multiple scattering of the probing light source, (ii) optical pumping effects initiated by the probe or MOT lasers, and (iii) the influence of the finite bandwidth of the probe laser. Each of these effects has been quantitatively ignored in earlier studies, since their role is not so crucial to calculations of the basic characteristics of the CBS process. Of particular interest is the influence of atomic motion and internal polarization variables on the spectral variations of the CBS enhancement. We point out that to properly account for these factors, it is necessary to consider the influence of the mean field on both attenuation and dispersion of the multiply scattered light and to include also the anisotropic Green's function for light propagating along a chain of scatterers. As is seen in the following section, inclusion of some such effects, in isolation or combination, may well be essential to better agreement between experimental and theoretical results.

Finally we emphasize that the simulations are made for conditions quite close to those in the experiment. These conditions include sample size, temperature, shape, and density and the characteristic intensity of the CBS laser beam. These conditions are such that cooperative scattering may be neglected and such that saturation of the atomic transition is also negligible. In simulations of thermal effects and of the influence of atomic magnetization on the coherent backscattering enhancement, more severe conditions are used in order to illustrate possible range of influence of the effects.

\section{EXPERIMENTAL AND THEORETICAL RESULTS}

In this section we present experimental and theoretical results associated with backscattering of near-resonance radiation from ultracold atomic ${ }^{85} \mathrm{Rb}$. First we present experimental measurements of the spectral variation of the coherent backscattering enhancement, in a range of approximately $\pm 6 \mathrm{MHz}$, as a function of detuning from the $F=3 \rightarrow F^{\prime}=4$ hyperfine transition. These results are directly compared to theoretical simulations made with inclusion of the influence of off-resonant hyperfine transitions and considering an ultracold sample not at absolute zero. Second, we present simulations of several effects which should generally be considered when modeling coherent backscattering from ultracold atomic vapors.

\section{A. Spectral variation of the CBS enhancement: Experimental results}

Measurements of the variation of the coherent backscattering enhancement with detuning of the CBS laser, in a

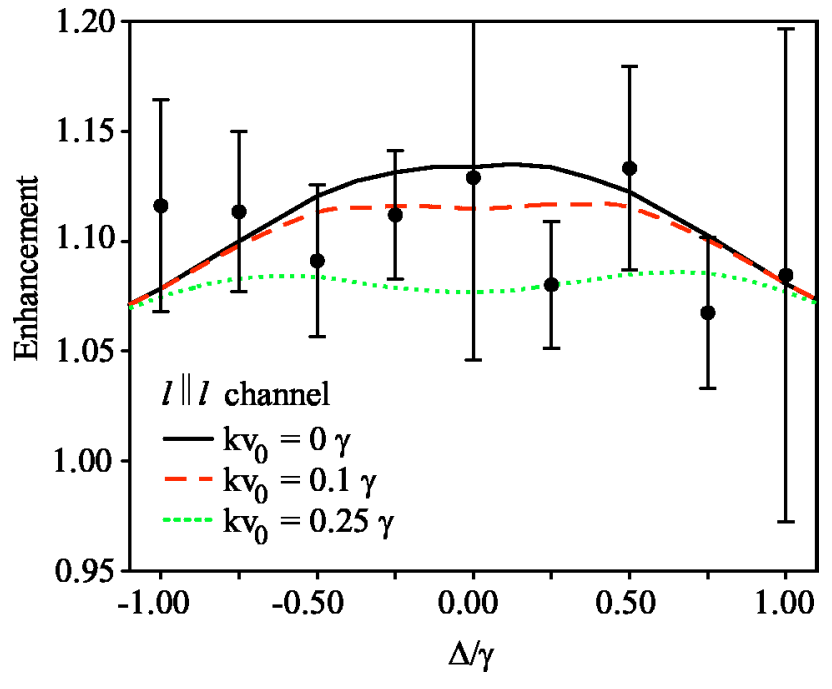

FIG. 3. Comparison of experimental and theoretical enhancement spectra in the $l \| l$ polarization channel. Theoretical spectra show modification by Doppler broadening, which is varying from $k v_{0}=0$ to $k v_{0}=0.25 \gamma$, in an ensemble of ${ }^{85} \mathrm{Rb}$ atoms having a peak density of $n_{0}=1.6 \times 10^{10} \mathrm{~cm}^{-3}$ and a Gaussian radius $r_{0}=1 \mathrm{~mm}$.

$\pm 6 \mathrm{MHz}$ range around the $F=3 \rightarrow F^{\prime}=4$ hyperfine transition, are shown in Figs. 3-6. The measurements have a typical uncertainty on the order of $2 \%$, this being due to a combination of statistical uncertainty due to counting statistics in the spatial intensity measurement, but also an estimated uncertainty due to the cone fitting procedure, as described previously [10]. In addition, there is residual noise in the spatial distribution of backscattered light due to speckle in the $l \| l$ and $h \perp h$ channels; slight variations in speckle appearing in background scattered light from run to run do not completely average to zero. This effect is responsible for the somewhat larger fluctuations in the extracted enhancement factors for these two polarization channels. There is a small systematic reduction of the peak enhancement due to the finite spatial resolution of the backscattering polarimeter, an effect which arises from smoothing of the nearly-cusp-shaped CBS cone

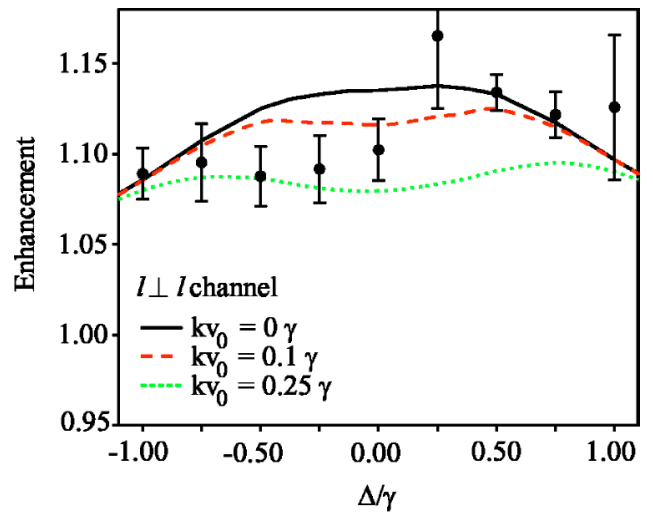

FIG. 4. Comparison of experimental and theoretical enhancement spectra in the $l \perp l$ polarization channel. Theoretical spectra show modification by Doppler broadening, which is varied from $k v_{0}=0$ to $k v_{0}=0.25 \gamma$, in an ensemble of ${ }^{85} \mathrm{Rb}$ atoms having a peak density of $n_{0}=1.6 \times 10^{10} \mathrm{~cm}^{-3}$ and a Gaussian radius $r_{0}=1 \mathrm{~mm}$. 


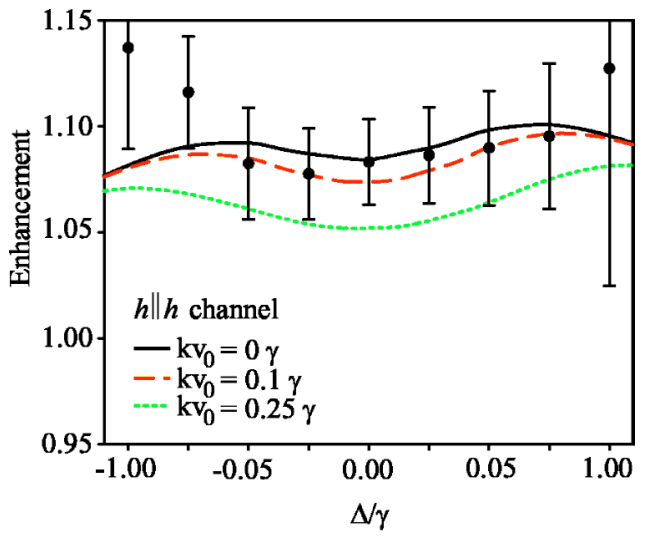

FIG. 5. Comparison of experimental and theoretical enhancement spectra in the helicity-preserving $(h \| h)$ polarization channel. Theoretical spectra show modification by Doppler broadening, which is varied from $k v_{0}=0$ to $k v_{0}=0.25 \gamma$, in an ensemble of ${ }^{85} \mathrm{Rb}$ atoms having a peak density of $n_{0}=1.6 \times 10^{10} \mathrm{~cm}^{-3}$ and a Gaussian radius $r_{0}=1 \mathrm{~mm}$.

near its peak by the finite spatial resolution of the instrument. This is accounted for by using a Lorentzian model of the spatial response and the CBS cone, which allows an estimate of the amount of reduction. Justification for this is made by the fact that the spatial variation of the simulated cones is to a good approximation described by a Lorentzian, as is the measured spatial response of the experimental apparatus. In our case, accounting for this effect amounts to a maximum decrease in the peak enhancement $\sim 0.01$ in the enhancement for the narrowest cones, which appear in the $h \| h$ data. This estimated correction is not made to the data in Figs. 3-6. On the scale of the figures, there is negligible uncertainty in the detuning measurements.

Also shown in the figures are simulations of the enhancement for several different values of the average Doppler shift of the atoms, measured in units of the natural spectral width

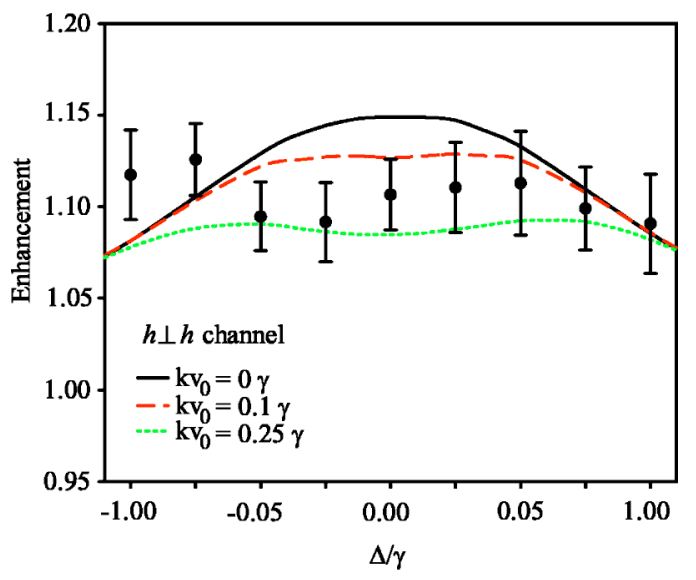

FIG. 6. Comparison of experimental and theoretical enhancement spectra in the helicity-nonpreserving $(h \perp h)$ polarization channel. Theoretical spectra show modification by Doppler broadening, which is varied from $k v_{0}=0$ to $k v_{0}=0.25 \gamma$, in an ensemble of ${ }^{85} \mathrm{Rb}$ atoms having a peak density of $n_{0}=1.6 \times 10^{10} \mathrm{~cm}^{-3}$ and a Gaussian radius $r_{0}=1 \mathrm{~mm}$. $\gamma$. Accounting for the uncertainty in the measured enhancements, there is very good agreement between the experimental data and the simulations assuming no influence of dynamical heating of the vapor. Although there are suggestive differences between the measurements and the simulations at $k v_{0}=0$, the measurement uncertainties are too large to make definitive conclusions at this point.

\section{B. Influence of dynamical heating}

The initial temperature of the atomic ensemble is $\sim 50 \mu \mathrm{K}$, which makes negligible any possible spectral manifestations caused by atomic motion. However, during the interaction time, the probe light produces a certain mechanical action on the atoms. The radiation force associated with the probe light can accelerate the atoms and heat them to temperatures where the Doppler broadening and shift can become comparable with the natural linewidth. It is important to recognize that the initial scattering event transfers momentum from the CBS laser to the atomic ensemble, but that subsequent scattering of the light deep within the sample is more nearly isotropic, resulting in some effective heating of the atoms during the CBS data-taking phase. Although the dynamical process is complex and is currently under study, we present here a short discussion of this process by comparing several scanning spectra averaged over an equilibrium Maxwell distribution of atom velocities - that is, for different temperatures and different Doppler widths, respectively. Momentum transfer from the CBS laser to the MOT ensemble also produces an average drift velocity of the atomic cloud. However, because initial light scattering of the CBS beam occurs in the vicinity of one absorption depth on the front boundary of the sample, this tends to produce a mixing of the accelerated atoms with cooler ones deeper inside the MOT. The entire process is complex, even when ultracold collisions between the atoms are ignored. In the present calculations, we include the average heating of the atomic sample, but ignore any average Doppler shifts of the spectral dependencies into the blue wing with respect to the laser frequency $\omega_{L}$

In addition to the experimental data, there are also shown in Figs. 3-6 calculated spectral variations of the enhancement factor for all four polarization channels. In the graphs, the velocity is indicated as a fraction of the natural width of the atomic transition, $k v_{0}\left(v_{0}=\sqrt{2 k_{B} T / m}\right.$ is the most probable velocity in the atomic ensemble). It is seen from these graphs that the shape of the spectra becomes significantly modified, even for an average Doppler breadth of $0.1 \gamma$, where $\gamma$ is the natural atomic width. Similar results are also obtained in the linear polarization channels. The overall trend in the calculations suggests that dynamical heating can have a significant effect on the measured enhancement values in coherent backscattering experiments. Of particular interest is the helicity preserving channel (Fig. 5). Unique to this case is a spectral asymmetry in the enhancement, even for no atomic motion, for moderate detunings away from exact resonance. As described in a previous report [15], the asymmetry is due to the nonnegligible influence of far-off-resonance hyperfine transitions on the coherent backscattering enhancement. In the fol- 

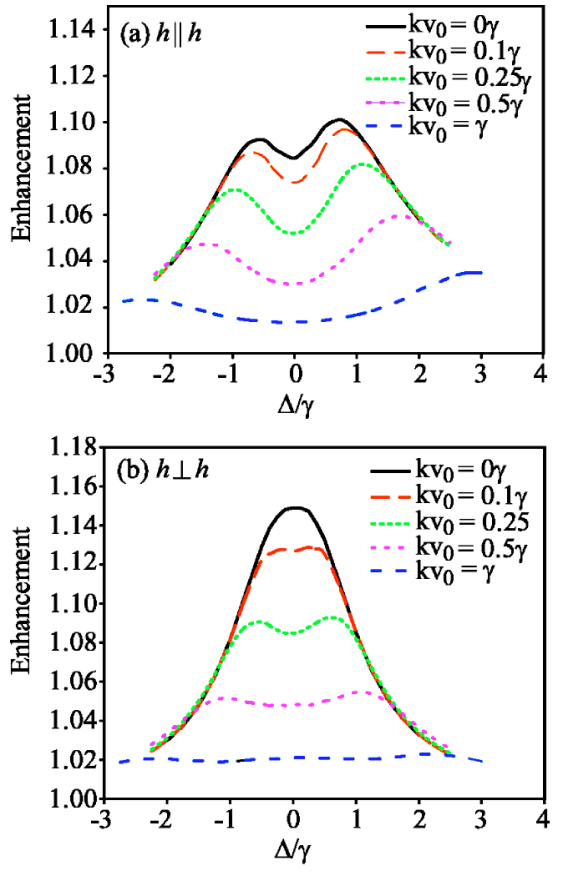

FIG. 7. Scanning spectra of CBS enhancement of (a) $h \| h$ and (b) $h \perp h$ polarization channels. Spectra are shown for an average Doppler broadening varying from $k v_{0}=0$ to $k v_{0}=0.25 \gamma$, in the ensemble of ${ }^{85} \mathrm{Rb}$ atoms with $n_{0}=1.6 \times 10^{10} \mathrm{~cm}^{-3}$ and $r_{0}=1 \mathrm{~mm}$.

lowing section, we see that a much larger enhancement increase at greater detunings can also arise from dynamical (or static) magnetization of the vapor along the direction of propagation of the CBS laser beam.

We finish this section by presenting for completeness theoretical results over a wider spectral range for the detuning dependence of the CBS enhancement. These are shown in Fig. 7(a) for the helicity-preserving channel and in Fig. 7(b) for the helicity-nonpreserving channel. The results show that there is some persistence of the CBS enhancement, even for an average Doppler broadening on the order of the natural linewidth of the atomic transition, and that this enhancement increases at larger detunings, before falling off at the largest offsets, when single scattering becomes dominant.

\section{Optical pumping effects}

Effects on coherent radiative transport in an atomic vapor will generally depend on the polarization of the incident light and on the nonzero ground-state multipoles in the atomic vapor. In our experimental arrangement, the atoms are confined to a magneto-optic trap, in which there exist generally spatially varying hyperfine multipoles. However, the MOT lasers are typically turned off for several ms before taking data in a coherent backscattering experiment, and residual macroscopic atomic polarization should be largely dissipated on that time scale. However, there can be hyperfine multipoles generated by the CBS laser itself, dynamically polarizing the vapor. The main argument why there is no optical pumping manifestation in the CBS process comes from the reasonable assumption that under not atypical experimental conditions the probe radiation is weak and characterized by a small saturation parameter. However, it is quite clear that in an ensemble of cold atoms the relaxation mechanisms in the ground state, which mainly are collisional, play a reduced role and become even negligible. Then, after each cycle of interaction with the polarized CBS light, the atomic ensemble can accumulate a certain degree of polarization, which may be either of an orientation or alignment type. Of particular interest to us here is when the incident radiation has definite helicity, which can magnetize the vapor along the CBS propagation direction. It is quite difficult to estimate precisely the actual dynamical spatial distribution, within the atomic cloud, of polarization generated during the whole interaction cycle. Therefore in this section we only qualitatively illustrate how the optical pumping effects can change a basic characteristic of the CBS process such as the enhancement factor.

Before proceeding to that discussion, we address a natural question to ask: how such an effect might be experimentally observable, given the multiple-light-scattering environment within the sample (which would tend to depolarize the vapor). One possibility is to modify existing experimental protocols such that, on the average, there is only one light scattering per atom in a given data taking cycle. Reoptically pumping the vapor between data taking cycles should sufficiently maintain the magnetization that the effect becomes observable. We suggest that a dynamical approach might also be effective. In this, if the CBS laser intensity is increased sufficiently, then optical pumping can occur simultaneously with multiple scattering. As single scattering should come to dominate at sufficiently high laser intensity, whereas multiple scattering of all orders is more effective at lower intensity, there could be a dynamical regime where there the trade-off between these two processes allows for some increase of enhancement to be measured.

Now let us return to discussion of the results of the simulation of the influence of atomic magnetization by considering probing of the atomic sample with positive helicity circular polarized radiation. Let us consider further that, due to optical pumping, the atoms become oriented only along the propagation direction of the light beam. In steady state, following a sufficiently long pumping time, if there is no relaxation in the ground state, the atoms should be concentrated in the Zeeman sublevel $F=3, M=3$; see Figs. 2 and 8. Of course, this is an idealized case which can never be precisely attained in reality, but such a model situation is convenient for illustrative purposes. The spectral variation of the enhancement factor for such an oriented ensemble is shown in Fig. 9 for the case of monochromatic probe radiation and for a Gaussian-type cloud with peak density $n_{0}$ $=1.6 \times 10^{10} \mathrm{~cm}^{-3}$ and radius $r_{0}=1 \mathrm{~mm}$.

The spectral variation of the enhancement in the helicitypreserving channel shows quite unusual behavior, in that there is no reduction of the CBS enhancement in the spectral wings. On the contrary, the enhancement factor approaches its maximal possible value of 2 . The limiting factor of 2 is normally associated with Rayleigh-type scattering on classical objects. But here we deal with Rayleigh-type scattering under approximately attainable, but not typical, quantum conditions. This result may be explained by a simple but fundamental property that in such a coherent atomic en- 


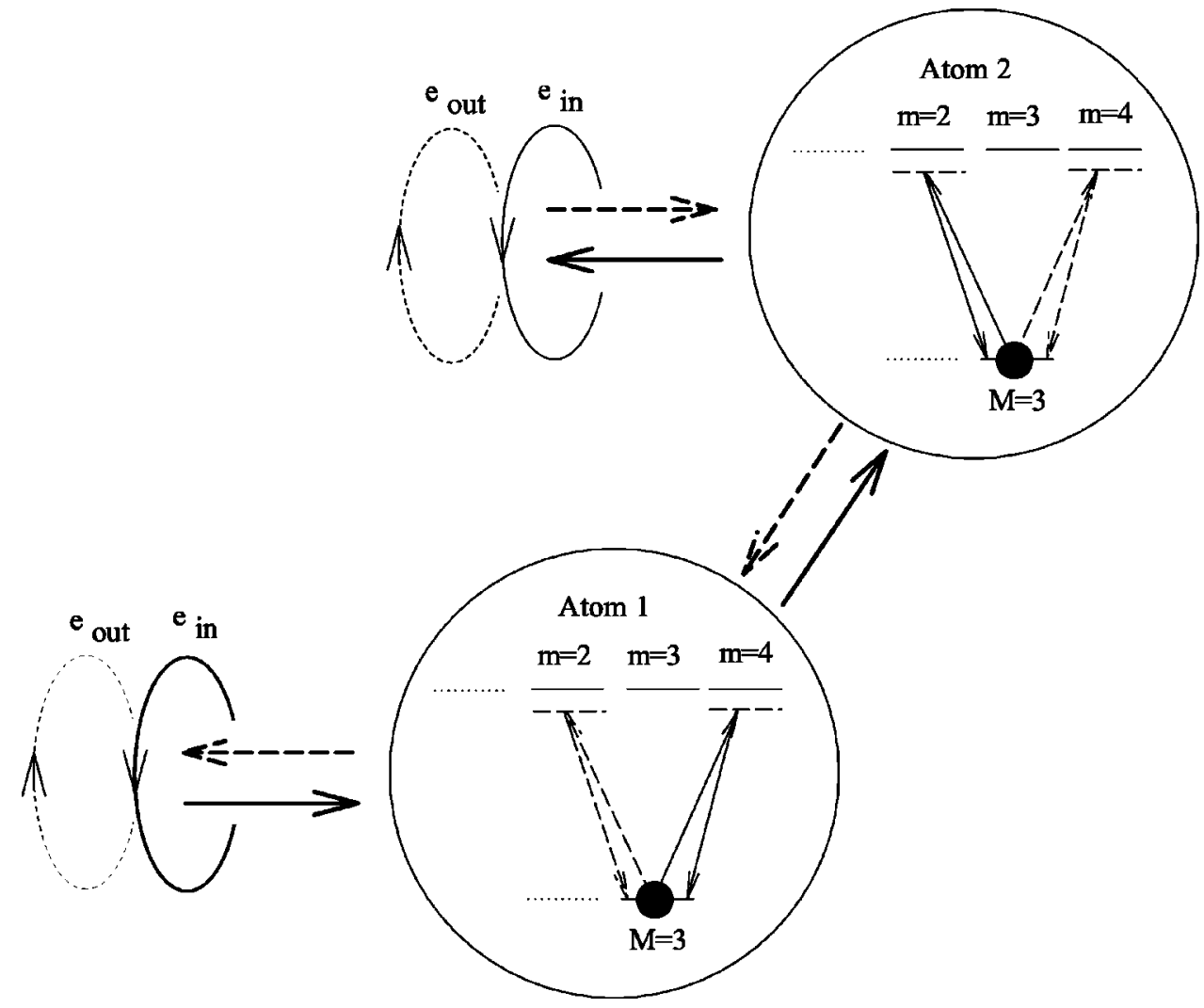

FIG. 8. Diagram explaining the CBS phenomenon for double scattering in an ensemble of oriented atoms of ${ }^{85} \mathrm{Rb}$ atoms. There is only one transition amplitude in the helicity-preserving channel, which leads to maximal enhancement of backward scattered light. The direct and reciprocal transitions and photon paths are shown by solid and dashed arrows, respectively.

semble there is no single-atom scattering of Raman-type radiation in the backward direction, which potentially could also be a source of backscattered light in the helicitypreserving channel. Moreover, the partial contribution of only double scattering on oriented atoms in optically thin sample causes the enhancement factor to take the maximum possible numerical value of 2 . This can be understood by turning to Fig. 8, where it is shown that there is only one channel or one product of the transition matrix elements con-

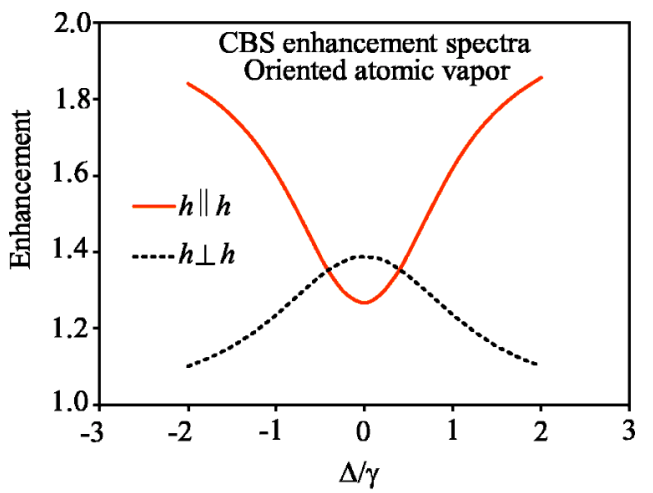

FIG. 9. Scanning spectra of enhancement for circularly polarized probe light in an ensemble of atoms of ${ }^{85} \mathrm{Rb}$ atoms with $100 \%$ orientation in the direction the light propagation. The spectra were calculated for a Gaussian-type atomic cloud with $n_{0}$ $=1.6 \times 10^{10} \mathrm{~cm}^{-3}$ and $r_{0}=1 \mathrm{~mm}$. tributing in the scattering amplitude of the double Rayleigh scattering - that is, in the helicity-preserving channel. These are ideal conditions to observe maximal enhancement in the CBS process. In higher orders there are several partial contributions and not all of them can interfere. This, as usual, leads to an essential reduction of the interference contribution to the total intensity of scattered light. Due to such a reduction, the enhancement factor considerably decreases in the spectral domain near the resonance scattering, as shown in Fig. 9. Thus in the wings of the helicity-preserving curve in the graph of Fig. 9 a unique situation is revealed when in an optically thin medium under special conditions the enhancement factor can increase to its maximal value.

The multiple scattering in the helicity-nonpreserving channel shows more ordinary behavior. The disappearance of CBS in the wings is caused by the dominating contribution of single-scattering events as far as the sample becomes optically thin. We point out that there is, for this polarization channel also, a certain increase of the maximal value of enhancement in comparing with a nonoriented atomic ensemble. Here, as in the linear polarization channels, we see that the optical pumping phenomenon leads to some quantitative but not qualitative changes in observation of the CBS process. However, the combined results of the numerical simulations presented in this and the previous section suggest that the experimental results may be essentially modified by the combined influence of thermal and optical pumping effects. 


\section{Finite bandwidth of the probe light spectrum}

In an experiment, the CBS probe laser operates ideally in a single-mode regime and its spectral bandwidth is much less than the natural relaxation rate of the atoms. But in reality the difference is not necessarily so great to completely ignore the spectral distribution of the laser radiation. Typically in our experiments on spectral scanning the sample consisting of rubidium atoms, which have a resonance line natural decay rate $\gamma \sim 5.9 \mathrm{MHz}$, the laser radiation has normally a bandwidth of less than $1 \mathrm{MHz}$. For the multiple-scattering process in higher orders, the scanned spectral profile of the sample is formed as a successive overlap of individual profiles per scattering event; the effective output shape reveals a much narrower spectral variance than $\gamma$. Thus the bandwidth of the laser mode can become comparable with the spectral inhomogeneity in the sample spectrum associated with partial contributions of the higher scattering orders.

This can be quantitatively discussed with the following model of a quasimonochromatic single-mode laser radiation. To define the basic parameters we approximated the assumed homogeneously broadened spectrum of the CBS laser by a Lorentzian profile

$$
I(\omega)=I \frac{\gamma_{L}}{\left(\omega-\omega_{L}\right)^{2}+\left(\gamma_{L} / 2\right)^{2}},
$$

where $\omega_{L}$ and $\gamma_{L}$ are the carrier frequency and spectral bandwidth of the laser radiation, respectively. $I$ is the total intensity of the incident laser radiation. The spectrum obeys the normalization condition

$$
I=\int_{-\infty}^{\infty} \frac{d \omega}{2 \pi} I(\omega),
$$

where in the quasimonochromatic approximation there is no difference between 0 and $-\infty$ in the lower limit of this integral. The basic idea in this case is that, in comparison with purely monochromatic radiation, the spectral response of the initially symmetric but broadened input spectral profile (1) should be significantly distorted by the sample due to effects of multiple scattering.

In Fig. 10 we show, in the helicity-preserving channel, the output spectral response in the backward direction when the input circularly polarized laser radiation, modeled by the spectrum of Eq. (1) with $\omega_{L}=\omega_{0}+1.5 \gamma$ and $\gamma_{L}=\gamma / 6$, is tuned in the blue wing of the $F=3 \rightarrow F^{\prime}=4$ optical transition of ${ }^{85} \mathrm{Rb}$ with resonant frequency $\omega_{0}$. The first graph plotted in Fig. 10(a) shows the distortion of the input Lorentzian profile for the total ladder and interference contribution, the intensity being normalized to the total output intensity of the CBS light. In turn, the second graph depicted in Fig. 10(b) shows the distortion of the interference term only. This term is normalized according to the corresponding enhancement factor $X_{\mathrm{EF}}$ [to $\left(X_{\mathrm{EF}}-1\right) / X_{\mathrm{EF}}$. Both dependences relate to the same helicity-preserving channel. It is clearly seen that the output spectral profile becomes asymmetric because of the influence of resonance scattering near the atomic transition in higher orders of multiple scattering. It may be less obvious, but there is also a small but not negligible difference between the
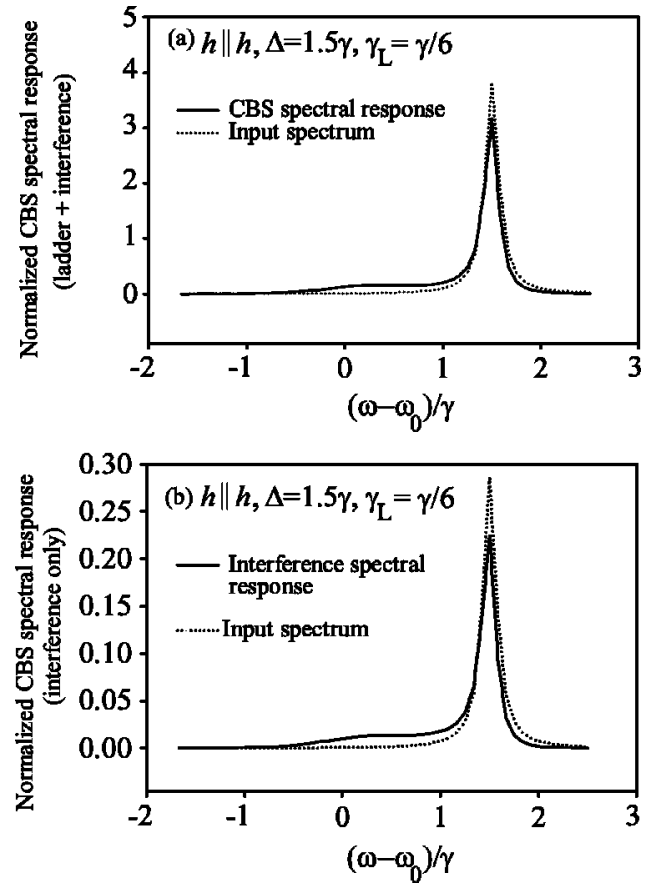

FIG. 10. The output spectral response of the CBS light when the input circularly polarized laser radiation, modeled by Lorentzian spectrum (1) with $\omega_{L}=\omega_{0}+1.5 \gamma$ and $\gamma_{L}=\gamma / 6$, is tuned in the blue wing of the $F=3 \rightarrow F^{\prime}=4$ optical transition of ${ }^{85} \mathrm{Rb}$. The first graph (a) shows the distortion of the input Lorentzian profile (dotted curve) for the total ladder and interference contribution; it is normalized to the total output intensity of the CBS light. The second graph (b) shows the distortion for the interference term only; it is normalized according to the corresponding enhancement factor. Both the dependences relate to the same helicity-preserving channel.

two spectral dependences, which explains why in our experiment the spectral probe of the sample with scanning carrier frequency $\omega_{L}$ near the resonance can be sensitive to the spectral bandwidth of the CBS laser.

The influence of this effect is illustrated, for the helicitypreserving and -nonpreserving channels, in Figs. 11 and 12. There it is shown that the spectra of the total intensity and of the enhancement factor, generated by scanning of the frequency $\omega_{L}$, reveals different spectral behavior, particularly in the wings of scanned profiles. These are calculated results for the helicity polarization channels, but similar behavior takes place for the linear polarization channel. At first sight this spectral divergence appears as a rather weak effect, but we believe it should not be ignored in a precise comparison of the experimental data with numerical simulations. Particularly, it can be important in a realistic estimation of the background, since such a spectral washing in the probe radiation response can be important in the interpolation procedure of the CBS cone to its wing. Indeed, the higher orders of multiple scattering contribute to the formation of the central portion of the CBS cone, but the role of second-order scattering is more important in its wings. As we see for large spectral detunings, the correct estimation of the enhancement factor in higher orders of multiple scattering is rather sensitive to the spectral distribution of the probe radiation. 

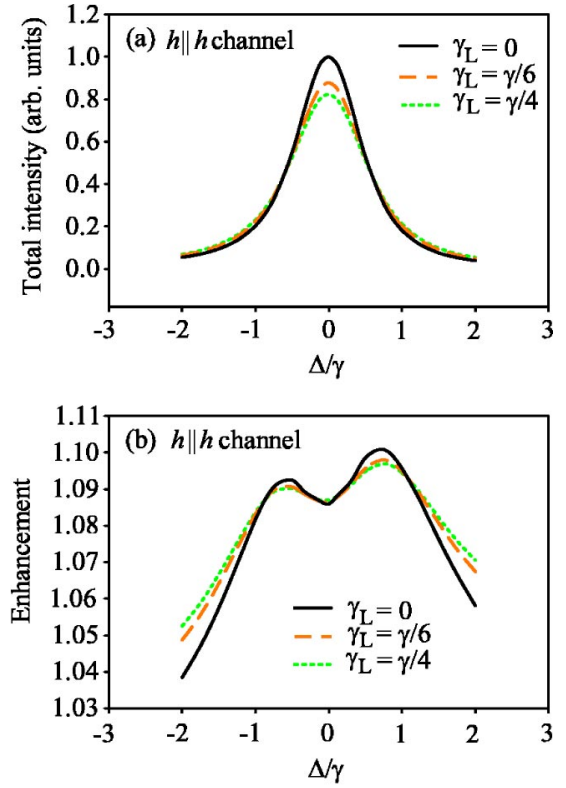

FIG. 11. Scanning spectra of the intensity (a) and of the enhancement factor (b) in the helicity-nonpreserving channel for the quasimonochromatic laser radiation, with $\gamma_{L}=\gamma / 4, \gamma / 6$, 0 . The spectra were calculated for a Gaussian-type atomic cloud of ${ }^{85} \mathrm{Rb}$ atoms with $n_{0}=1.6 \times 10^{10} \mathrm{~cm}^{-3}$ and $r_{0}=1 \mathrm{~mm}$.

\section{SUMMARY}

A joint theoretical and experimental study of spectral variations in the coherent backscattering enhancement factor, for a very narrow band resonance system, has been reported. Experimental data taken over a range of two atomic natural widths about direct atomic resonance suggest spectral variations in the peak value of the CBS enhancement. Simulations indicate that the combined influence of heating of the atomic ensemble and optical pumping of the Zeeman sublevels in the $F=3$ ground level during the coherent backscattering data taking phase can also significantly influence the enhancement. Simulations of the CBS process examined the influence of atomic motion, in a thermal equilibrium model, on the spectral variation of the enhancement factor. A model case of magnetization of the vapor due to optical pumping was also considered. The simulations, which considered the influence of atomic magnetization, predicted a remarkable
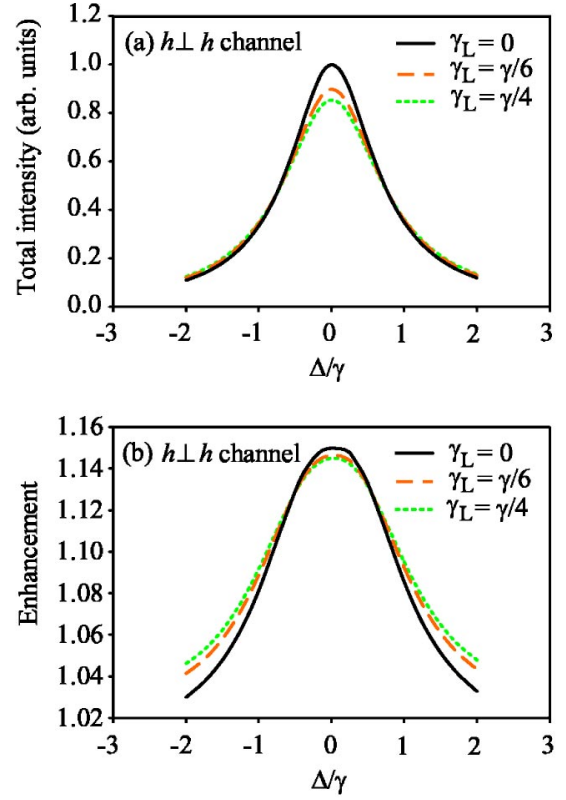

FIG. 12. Scanning spectra of the intensity (a) and of the enhancement factor (b) in the helicity-preserving channel for quasimonochromatic laser radiation with $\gamma_{L}=\gamma / 4, \gamma / 6,0$. The spectra were calculated for a Gaussian-type atomic cloud of ${ }^{85} \mathrm{Rb}$ atoms with $n_{0}=1.6 \times 10^{10} \mathrm{~cm}^{-3}$ and $r_{0}=1 \mathrm{~mm}$.

result; the classical CBS maximum enhancement of 2 could be closely approached for a strongly magnetized atomic sample. Finally, it was shown, by simulation of the influence of the spectral bandwidth of the CBS probe laser, that even a quite small laser linewidth in comparison to the natural width of the atomic transition can influence significantly the CBS enhancement in the wings of the atomic resonance line.

\section{ACKNOWLEDGMENTS}

We acknowledge informative discussions with Robin Kaiser. Financial support for this research was provided by the National Science Foundation (Grant Nos. NSF-PHY0099587 and NSF-INT-0233292), by the North Atlantic Treaty Organization (Grant No. PST-CLG-978468), by the Russian Foundation for Basic Research (Grant No. 01-0217059), and by INTAS (Grant No. INFO 00-479). D.V.K. would like to acknowledge financial support from the Delzell Foundation, Inc.
[1] M. I. Mishchenko, Astrophys. J. 411, 351 (1993).

[2] New Aspects of Electromagnetic and Acoustic Wave Diffusion, edited by the POAN Research Group, Springer Tracts in Modern Physics, Vol. 44 (Springer-Verlag, New York, 1998).

[3] Ping Sheng, Introduction to Wave Scattering, Localization, and Mesoscopic Phenomena (Academic, San Diego, 1995).

[4] Ad Lagendijk and B. A. van Tiggelen, Phys. Rep. 270, 143 (1996).

[5] P. W. Anderson, Phys. Rev. 109, 1492 (1958).
[6] D. S. Wiersma, P. Bartolini, Ad Lagendijk, and R. Righini, Nature (London) 390, 671 (1997).

[7] A. A. Chabanov, M. Stoytchev, and A. Z. Genack, Nature (London) 404, 850 (2000).

[8] G. Labeyrie, F. De Tomasi, J-C. Bernard, C. A. Muller, C. A. Miniatura, and R. Kaiser, Phys. Rev. Lett. 83, 5266 (1999).

[9] G. Labeyrie, C. A. Muller, D. S. Wiersma, Ch. Miniatura, and R. Kaiser, J. Opt. B: Quantum Semiclassical Opt. 2, 672 (2000). 
[10] P. Kulatunga, C. I. Sukenik, S. Balik, M. D. Havey, D. V. Kupriyanov, and I. M. Sokolov, Phys. Rev. A (to be published).

[11] Y. Bidel, B. Klappauf, J. C. Bernard, D. Delande, G. Labeyrie, C. Miniatura, D. Wilkowski, and R. Kaiser, Phys. Rev. Lett. 88, 203902 (2002).

[12] J. Ishimaru and Yu. Kuga, J. Opt. Soc. Am. A 1, 813 (1984).

[13] P. E. Wolf and G. Maret, Phys. Rev. Lett. 55, 2696 (1985).

[14] M. P. VanAlbada and A. Lagendijk, Phys. Rev. Lett. 55, 2692 (1985).

[15] D. V. Kupriyanov, I. M. Sokolov, P. Kulatunga, C. I. Sukenik, and M. D. Havey, Phys. Rev. A 67, 013814 (2003).

[16] Guillaume Labeyrie, Dominique Delande, Cord A. Mueller, Christian Miniatura, and Robin Kaiser, Europhys. Lett. 61,
327 (2003).

[17] Guillaume Labeyrie, Dominique Delande, Cord A. Mueller, Christian Miniatura, and Robin Kaiser, Phys. Rev. A 67, 033814 (2003).

[18] T. Jonckheere, C. A. Muller, R. Kaiser, Ch. Miniatura, and D. Delande, Phys. Rev. Lett. 85, 4269 (2000).

[19] G. Labeyrie, C. Miniatura, C. A. Müller, O. Sigwarth, D. Delande, and R. Kaiser, Phys. Rev. Lett. 89, 163901 (2002).

[20] Robin Kaiser (private communication).

[21] Harold J. Metcalf and Peter van der Straten, Laser Cooling and Trapping (Springer, New York, 1999).

[22] Th. M. Nieuwenhuizen, A. L. Burin, Yu. Kagan, and G. V. Shlyapnikov, Phys. Lett. A 184, 360 (1994). 